\title{
Description and Reliability of Rigo Classification of Adolescent Idiopathic Scoliosis Associated with Brace Therapy
}

\author{
Junze Chen ${ }^{1}$, Bagen Liao ${ }^{2, *}$ \\ ${ }^{1}$ Department of Orthopaedics, Guangzhou The People's Hospital of Baiyun District, Guangzhou, China \\ ${ }^{2}$ Department of Sports Medicine, Guangzhou Sports University, Guangzhou, China
}

Email address:

961262497@qq.com (Junze Chen), bagen2015@163.com (Bagen Liao)

${ }^{*}$ Corresponding author

\section{To cite this article:}

Junze Chen, Bagen Liao. Description and Reliability of Rigo Classification of Adolescent Idiopathic Scoliosis Associated with Brace Therapy. International Journal of Clinical and Experimental Medical Sciences. Vol. 5, No. 4, 2019, pp. 53-57. doi: 10.11648/j.ijcems.20190504.11

Received: July 9, 2019; Accepted: August 10, 2019; Published: August 23, 2019

\begin{abstract}
Objectives To develop the Rigo classification system for scoliosis brace design and treatment and to compare the reliability and reproducibility of the Rigo classification system for scoliosis within and across observers related to orthopedic treatment. Methods The imaging data of 136 cases of adolescent idiopathic scoliosis were collected, and a Rigo classification system was established to determine the specific corrective principles and methods needed for the effective design and manufacturing of braces. Three observers, one spine surgeon, one imaging surgeon and one orthopedic brace surgeon, diagnosed and classified 136 AIS patients. The collected data were used to analyze the reliability across different observers and the repeatability within the same observer, and kappa values were calculated to confirm the consistency. Results The kappa value of the classification results of the spine surgeons and radiologists was 0.936 , that of the spine surgeons and brace surgeons was 0.901 , that of the imaging surgeons and brace surgeons was 0.886 , the average repeatability was $90 \%$, and the average Kappa was 0.887. Conclusion The Rigo classification system for scoliosis brace therapy has good practicability and consistency. The reliability of the Rigo classification system is acceptable both within and across observers. The system is suitable for clinical applications.
\end{abstract}

Keywords: Adolescent Idiopathic Scoliosis, Rigo Classification, Reliability Test, Brace Therapy

\section{Introduction}

Idiopathic scoliosis is a three-dimensional deformity of the spine and trunk induced by multiple factors. Idiopathic scoliosis may occur in healthy children when they are growing quickly. Growth and development primarily occur during adolescence, and deformities may occur during this period [1]. There are many classification systems for adolescent idiopathic scoliosis (AIS), such as the Lenke and PUMC classification systems, but these classification systems are mainly used to guide spinal surgery. They are not suitable for AIS patients who need conservative treatment in the early stage, and orthopedic brace treatment is considered as clinical treatment. The Milwaukee brace, Boston brace, Wilmington brace, Cheneau brace and Charleston lateral brace are important types of braces for mild to moderate AIS.
Hueter-Volkmann's law states that the use of a brace can reduce the growth of cartilage endplates in scoliosis and accelerate growth on the concave side, thereby improving or preventing the progression of the deformity $[2,3]$. At present, the King classification system, or the "three arcs" and "four arcs" scoliosis classification system, is still used in the preparation and adjustment of Xenopus braces after orthopedic surgery; however, foreign scholars focus on and commend the Rigo classification system more recently developed by Lehnert-Schroth, which is an extension of the "three arcs" and "four arcs" classification system. In addition to the Rigo classification system, the deformity appearance and an imaging diagnosis are used to guide the design and preparation of AIS Snoop braces, as well as review and adjust the braces worn by AIS patients for continuous orthopedic treatment [4]. This study used the Rigo classification system 
to classify AIS types and validated the reliability of the Rigo classification system and brace therapy with this system to provide an experience summary for the nonsurgical treatment of AIS patients.

\section{Materials and Methods}

\subsection{Clinical Data}

A database of 136 AIS patients was established. There were 18 males and 118 females, aged 11-14 years, with an average age of $(12.16 \pm 0.62)$ years. Three observers, one spine surgeon, one imaging surgeon and one orthopaedic brace surgeon, were used to diagnose and classify 136 patients by Rigo. All 136 AIS patients met the following criteria: age $>10$ years old; Risser sign: 0-2 grade; primary scoliosis Cobb angle 20-40 degrees, without any treatment before; female patients with end-of-menstruation or menstruation to less than one year.

\subsection{Method}

\subsubsection{Rigo Typing Standard}

According to the observation of clinical signs, it can be divided into three curves pattern (3C), four curves pattern (4C), non-three non-Four (N3N4) or single lumbar and thoracolumbar (L/TL), and clinical subtyping according to imaging criteria. The $3 \mathrm{C}$ type is divided into $\mathrm{A} 1, \mathrm{~A} 2$ and $\mathrm{A} 3$ subtypes; the $4 \mathrm{C}$ type is divided into $\mathrm{B} 1$ and $\mathrm{B} 2$ subtypes; the N3N4 type is divided into C1 and C2 subtypes; and the L/TL type is divided into E1 and E2 subtypes.

\subsubsection{Imaging Criteria}

International Scoliosis Research Society ( SRS) defines scoliosis as standing X-ray film, midline deflection of spine and Cobb angle $>10$ degrees, mainly chest bending, lumbar double bending, single lumbar bending, single thoracolumbar bending and so on. The apical vertebra is the most distant and horizontal vertebral body or intervertebral disc. The name of the horizontal determination curve of the apical vertebra is T2-T11 for thoracolumbar bending, T12-L1 for thoracolumbar bending and L2-L4 for lumbar bending. Center Sacral Line (CSL), Transitional Piont (TP), TP is located between the curve below the thoracic bend and the curve below the thoracic bend. TP is the transition point between the lower end vertebrae (LEV) and the next upper end vertebrae (UEV). And trunk force line. T1 is balanced or imbalanced on the CSL line, located on the convex or concave side of the chest. If the inclination of L4 and L5 to sacrum is in the same direction, the inclination of L4 lower end plate and L5 upper end plate is parallel, and negative inclination (-Counter-tilting); if the inclination of L4 and L5 to sacrum is not in the same direction (the sacrococcyx is inverse scoliosis), it is positive inclination (+Counter-tilting).

D-modifier is used to define structural scoliosis of the upper thoracic spine, "D-modifier" may exist in Rio A, B or C subtypes.

\subsubsection{Rigo Subtype Description}

i. Rigo $3 \mathrm{C}$ subtype
(1) Type A1 clinical signs: pelvis lateral to thoracic concave and outward projection; trunk imbalance, center of gravity in thoracic convex side, thoracic convex side leg bearing; If the pelvis does not move laterally, the sternum tends to move laterally to the thoracic concave. The rib protrusion on the back of the chest is longer, and the protrusion on the same side of the waist is also seen. X-ray features: single long thoracic curve (top vertebra in T19-T11) involved the upper segment of lumbar curve, L3 in the thoracic convex side was inclined, L4 in the horizontal or inclined to the thoracic convex side, L5 in the horizontal position; TP, T1 in the thoracic convex side, the entire spine in the CSL side; may be accompanied by proximal thoracic curve (top vertebra in T2-T4).

(2) Type A2 clinical signs: pelvis lateral to thoracic concave and protruding outwards; trunk unbalanced, center of gravity on thoracic convex side, chest convex side bearing legs; sternum lateral to thoracic convex side, if pelvis not lateral, sternum lateral to thoracic concave; thoracic back has a rib protrusion, no/or small lumbar protrusion on the opposite side. $\mathrm{X}$-ray features: main thoracic curvature (top vertebra at T5-T9), no or only upper lumbar curvature, L4-5 in horizontal position. TP and T1 were located on the thoracic convex side, and the whole vertebra was located on the side of CSL, accompanied by proximal thoracic curvature (the apex was in T2-T4).

(3) Type A3 clinical signs: the pelvis lateral to the thoracic concave and protrudes outwards; the trunk is unbalanced, the center of gravity is on the thoracic convex side and the thoracic convex side bears the weight of the legs; the sternum lateral to the thoracic convex side, if the pelvis does not move laterally, the sternum lateral to the thoracic concave; there is a rib peak on the back of the chest and a small lumbar protrusion. X-ray features: main thoracic curve (top vertebra in T5-T9), secondary lumbar curve (top vertebra in L2-4), L4-5 inclination (downward) to thoracic concave side (negative L4-5 inclination); TP, $\mathrm{T} 1$ located in thoracic convex side, the whole thoracic and lumbar spine in one side of CSL; may be accompanied by proximal thoracic curve (top vertebra in T2-T4).

ii. Rigo 4C subtype

(1) Type B1 clinical features: pelvis lateral to thoracolumbar protrusion and protrusion outward, sternum lateral to thoracic concave; trunk center of gravity at thoracic concave side, bearing weight of thoracic concave side foot; rib protrusion at thoracic back, contralateral lumbar or thoracolumbar protrusion; often accompanied by thoracic concave side foot valgus, hip rotation. X-ray features: double curvature, main thoracic curvature in T5-T9, lumbar curvature in L2-4 or thoracolumbar curvature in T12-L1, positive L4-L5 inclination (lumbosacral 
curvature). TP was on the concave side of the chest and $\mathrm{T} 1$ was on the concave side of the chest. The proximal thoracic curvature vertebra was at T2-T4.

(2) Type B2 clinical features: pelvis lateral to thoracic convex, protruding outward, sternum lateral to thoracic concave; trunk center of gravity on thoracic concave side, bearing weight on thoracic concave side foot; back with mild rib peak, thoracolumbar protrusion obvious; often accompanied by thoracic concave side of foot valgus, hip rotation. X-ray features: main thoracolumbar curvature (top vertebra at T12-L1) +minor thoracic curvature, top lumbar curvature at L2 (or L3) or low TL curvature (L1), positive L4-L5 inclination (lumbosacral inverted scoliosis); TP at the thoracic concave side, T1 at the thoracic concave side; may be accompanied by proximal thoracic curvature (top vertebra at T2-T4).

iii.Rigo N3N4 subtype

(1) Type C1 clinical signs: pelvic neutrality, trunk balance; prominent rib protrusion, lumbar straight line. X-ray features: single thoracic curvature, the top of thoracic curvature in T8-T9, no obvious lumbar curvature; T1 and TP in CSL, L4-L5 in horizontal position. It may be accompanied by proximal thoracic curvature (apical vertebra at T2-T4).

(2) Type C2 clinical signs: pelvic neutrality, trunk balance; significant rib peak, contralateral lumbar prominence. X-ray features: main thoracic curve + lumbar curve; T1 and TP on CSL, negative L4-L5; may be accompanied by near thoracic curve (apical vertebra in T2-T4).

iv. Rigo L/TL subtype

(1) Type E1 clinical signs: pelvis shifted to lumbar concave side; center of gravity of unbalanced trunk was on lumbar convex side; prominent lumbar protrusion. X-ray features: single lumbar curve, no thoracolumbar curve, lumbar curvature apex in L1-L2; T1 and TP in the lumbar convex side, the entire thoracolumbar spine in the CSL side.

(2) Type E2 clinical signs: pelvis shifted to lumbar concave side, trunk unbalanced, center of gravity at lumbar convex side, obvious thoracolumbar protrusion. X-ray features: single thoracolumbar curve, the apex at $\mathrm{T} 12 ; \mathrm{T} 1$ and $\mathrm{TP}$ at the lumbar convex side, the whole thoracolumbar spine at the CSL side.

\subsection{Study on Reliability and Repeatability of Typing}

One spine surgeon, one imaging surgeon and one orthopaedic orthopaedic orthopaedic orthopaedic orthopaedic orthopaedic surgeon were selected for typing training: definition of scoliosis, Cobb angle measurement of scoliosis, clinical observation and imaging standards of Rigo classification. After passing the X-ray test, 136 cases were selected according to the random number table method and then numbered. After being measured and classified by the above three persons, the results of the observer's classification were collected and the reliability was analyzed. Twenty weeks later, the results were typed again and analyzed for repeatability.

\subsection{Statistical Methods}

The collected data were analyzed by SPSS19.0 software and the Kappa coefficient was calculated. The values of Kappa are between $[-1,+1]$, and the values of kappa are less than 0.20 , which indicates that the consistency is very poor; the values of kappa are between 0.21 and 0.40 , and the consistency is acceptable; the values of kappa are between 0.41 and 0.60 , and the consistency is medium; the values of kappa are between 0.61 and 0.80 , and the consistency is good; and the values of kappa are between 0.81 and 1.00, and the consistency is very good.

\section{Results}

The study was designed to test the intra-observer and the inter-observer reliability of the radiological criteria. Three different observers, one medical doctor (A), one A radiologist (B) and one orthotist (C) were asked to classify a set of all AP radiographs taken from the database, including all the scoliosis types. Researchers had to classify the whole set for a second time 78 hours after the first trial. The X-rays were presented to each observer in a printed version with no pre-determined marks. For the intra-observer study the whole set of X-rays was printed twice and ordered in a different way. Inter-observer Kappa values were calculated three times (A versus $B, B$ versus $C$ and $A$ versus $C$ ), as well as the mean value, with an acceptance of $>0.70$. Intra-observer Kappa value was calculated (A versus A) with an acceptance of $>0.70$ Considering the correlation between type and brace design we defined six categories: A1 type, A2 + A3 types, B1 + B2types, $\mathrm{C} 1+\mathrm{C} 2$ types, E1 and E2. The upper structural curve was not considered because the need to use the ' $D$ modifier' in the brace design is closely associated with the presence of an upper rib hump observed during the direct examination of the patient in forward bending. The mean Kappa value in the inter-observer test was 0.89 . The Kappa value in the intra-observer test was 0.91 . Both inter- and intra-observer mean Kappa values were over the acceptance value of 0.70 . The highest intra- and inter-observer agreement was noted in types B1 + B2, E1 and E2. Thus, the socalled 'four curves' scoliosis pattern or 'true double' as well as single lumbar and thoracolumbar are easy to diagnose by using these radiological criteria.

\section{Discussion}

Adolescent idiopathic scoliosis (AIS) [5, 6], or delayed scoliosis, is a disease of abnormal curvature and rotation of the spine in the coronal plane accompanied by vertebral rotation, which creates an arc-like shape in the patient's spine. The etiology of AIS is still unclear. There are many classification systems for AIS, but most of them, such as the widely used Lenke and PUMC classification systems, are used to guide the 
selection of surgical procedures for severe AIS. The Lenke classification system, which is used to guide spinal surgery, has proven to be more reliable than the King classification system and has been used for spinal surgery [7]. de Mauroy et al. reported technical specifications of brace structures according to different Lenke types, but the Lenke classification system is relatively complicated when it is used to make and trim Cheneau braces and their derivatives [4]. The definition of structural scoliosis is controversial. Clinical observations are inconsistent. $[9,10]$ There are often different opinions on the design of braces. Correction effects differ by brace type. Qiu Guixing and other researchers have developed the PUMC classification system that includes a better selection of surgical methods and range of fusion levels and provides clinical guidance [11]. However, there is no consensus on the correction and classification of scoliosis braces. The Lenke and PUMC classification systems are seldom used in brace design, but brace orthopedic treatment is considered to be an effective means to control the progression of moderate AIS. At present, the biomechanical research on braces in the treatment of AIS mainly focuses on two aspects: how to adjust the existing brace to improve its therapeutic effect and how to design a brace more in line with the biomechanical behavior of scoliosis correction. However, a series of problems, such as the direction and size of corrective forces exerted by braces, the specific relationship between the tension of the straps and the pressure of the pads [12], and the changes in posture and the adjustments of the braces, need to be further studied [13]. The Rigo classification system for scoliosis brace therapy has good practicability and consistency. This system includes all the common types of idiopathic scoliosis. It combines the coronal, sagittal and axial deformities of scoliosis for reference classification. It has high reliability and repeatability, and each classification has a corresponding correction method. It offers practical clinical guidance [14]. It is helpful for clinicians to obtain more accurate and reliable diagnostic criteria and achieve the most effective nonsurgical orthopedic treatment. The correction of scoliosis is far from being as simple as the correction of the Cobb angle. More attention should be paid to the balance of body and spine and the aesthetic evaluation of patients. The use of a reasonable classification system is very important to the design of braces and guides more effective nonsurgical orthopedic treatment. However, there is no consensus on the orthopedic classification of scoliosis braces. The Lenke and PUMC classification systems are seldom used in brace design. Lehnert-Schroth developed a classification system named "three arcs" and "four arcs" to guide the physical treatment of scoliosis. The terminology and diagnostic criteria are relatively simple. Cheneau adopted Lehnert-Schroth's terminology of "three-arc" and "four-arc" scoliosis models and developed "three-arc" scoliosis braces and "three-arc" scoliosis braces. The Rigo classification system is an extension of Lehnert-Schroth's three-arc and four-arc classification system. Rigo previously developed a classification system for Cheneau braces and their derivatives (RCB). The clinical standard is based on the Lehnert-Schroth description, and the imaging standard is used to make the braces that are designed and manufactured more suitable according to biomechanical principles [4]. The classification system, which uses the SRS definition of scoliosis and the location of the TP of T1 in CSL on X-ray film to help determine the classification and trunk line of force, is relatively concise and evidence-based. For example, the "three arcs" classification represents types of AIS with a deviation to the right in the shape and appearance of the trunk, a left convex pelvic manifestation, and according to the TP of the vertebrae visible in X-ray images, a thoracic curve moves toward the convex side from T1 (the top thoracic vertebra); this type of AIS may be accompanied by a compensatory lumbar curve, and three apexes, A1, A2 and A3, can be distinguished. According to the appearance characteristics of a left trunk deviation and a right pelvic protrusion, a double curvature spanning thoracic and lumbar vertebrae is classified as "four arcs". With the use of X-ray imaging, the TP of T1 can be located on the concave side of the thoracic curvature, and the location of top vertebrae of the lumbar curvature is divided into B1 and B2 subtypes. The classification system emphasizes the above image evaluation index of coronal balance. By using the central axis and reference point, this method reduces the risk of misclassification and is used to guide the design and assembly of the AIS Snoop Brace [4]. When correcting AIS curves with the Senu brace, three mechanisms are combined: coronal three-point force correction, regional and local anti-rotation, and sagittal balance and physiological curve restoration; with these mechanisms, the static brace can produce the necessary torsion forces to achieve an ideal three-dimensional correction of scoliosis. The Rigo classification system includes a description of a proximal thoracic curvature. When designing braces according to the classification, attention should be paid to the balance of the pelvis and spine and an aesthetic evaluation of each patient. The corrective effect has been preliminarily verified [15]. In this study, reliability tests were carried out for three specialists who are closely related to the design and manufacturing of braces. The results show that the consistency is high, which is beneficial for clinical applications.

In this study, the "three arcs", "four arcs", "non-three", "non-four" and "single waist/single thoracolumbar bending" classifications are quite consistent. The following are some reasons for inconsistencies in determining classifications: first, the location of the apex causes inconsistencies between A2 and $\mathrm{A} 3$ and between $\mathrm{B} 1$ and $\mathrm{B} 2$; second, the location of the apex and the diagnosis of a compensatory thoracic curvature appear inconsistent between B1 and B2 and between E1 and E2. However, the number of experts and the size of the sample population included in the study is still small. For the D-modified upper thoracic curve, no single-column classification has been performed, and further research is needed to expand the sample size in the future. With a long-term follow-up of the corrective effect of Rigo type braces, the understanding of conservative treatment of scoliosis will be more profound and thorough. An appropriate 
classification system will provide an objective basis for clinical treatment and prognosis to effectively control the progression of scoliosis and achieve better corrective effects.

\section{Conclusion}

Rigo classification of scoliosis has been described as related to brace therapy, and its classification is based on clinical signs and radiology. When clinicians, radiologists and orthopaedic orthopaedics master Rigo classification, they will optimize clinical diagnosis, malformation classification and brace design for AIS patients more accurately. In clinical orthopaedic treatment, it will help to promote the non-surgical treatment of AIS patients and reduce the failure rate of orthopaedic treatment with braces.

\section{Funding}

Guangdong Science and Technology Project (2017A020220003).

\section{Competing Interests}

We have no competing interests.

\section{Research Ethics}

We received ethical approval from the ethic committee for examination and approval of Guangzhou The people's Hospital of Baiyun District to carry out our study. We received informed consent for the participants to participate in the study.

\section{References}

[1] Lonstein J, Bradford D, Winter R, Ogilvie J: Moe's textbook of scoliosis and other spinal deformities Philadelphia PA: WB Saunders, Third 1995.

[2] Chase AP, Bader DL, Houghton GR. The biomechanical effectiveness of the Boston brace in the management of adolescent idiopathic scoliosis [J]. Spine, 1989, 14 (6): 636642.

[3] Wong MS, Mak AF, Luk KD, et al. Effectiveness and biomechanics of spinal orthoses in the treatment of adolescent idiopathic scoliosis (AIS) [J]. Prosthet Orthot Int, 2000, 24 (2): 148-162.

[4] Manuel D Rigo, Mónica Villagrasa1, Dino Gallo. A specific scoliosis classification correlating with brace treatment: description and reliability. Scoliosis 2010 5: 1-11.

[5] Barton CB, Weinstein SL. Adolescent Idiopathic Scoliosis: Natural History [M] / Pathogenesis of Idiopathic Scoliosis. Springer, Tokyo, 2018: 27-50.

[6] Choudhry MN, Ahmad Z, Verma R. Adolescent idiopathic scoliosis [J]. Open Orthop J, 2016, 10 (11): 143.

[7] Lenke LG, Edwards CC, Bridwell KH: The Lenke classification of adolescent idiopathic scoliosis: how it organizes curve patterns as a template to perform selective fusions of the spine [J], Spine, 2003, 28: 199-207.

[8] D'Amato CR, Griggs S, McCoy B: Nighttime bracing with the Providence brace in adolescent girls with idiopathic scoliosis [J]. Spine, 2001, 26: 2006-2012.

[9] QIU Gui- xing, YU Bin, Norbert Ventura, et al. Application and analysis of King, Lenke and PUMC classifications of idiopathic scoliosis [J]. Chin J Orthop, March 2006, Vd. 26, No. 3: $145-150$.

[10] Richards BS, Sucato DJ, Konigsberg DE, et al. Comparison of reliability between the Lenke and King classification systems for adolescent idiopathic scoliosis using radiographs that were not premeasured [J]. Spine, 2003, 28: 1148-1157.

[11] QIU Gui-xing, ZHANG Jian-guo, WANG Yi-peng, et al. The PUMC operative classifyion system for idiopathic scoliosis. Chin J Orthop, January 2003, 23 (1): 1-9.

[12] Mac- Thiong JM, Petit Y, Aubin CE, et al. Biomechanical evaluation of the Boston brace system for the treatment of adolescent idiopathic scoliosis: relationship between strap tension and brace interface forces [J]. Spine, 2004, 29 (1): 26- 32.

[13] Clin J, Aubin CE, Parent S, et al. Comparison of the biomechanical 3D efficiency of different brace designs for the treatment of scoliosis using a finite element model [J]. Eur Spine J, 2010, 19 (7): 1169-1178.

[14] Qiu G, Zhang J, Wang Y, et al. A new operative classification of idiopathic scoliosis: a Peking Union Medical College method [J]. Spine, 2005, 30: 1419-1426.

[15] Manuel Rigo, and Mina Jelačić. Brace Technology Thematic Series: The 3D Rigo Chêneau-type Brace [J]. Scoliosis and Spinal Disorders 2017, 12 (10): 1-49. 